Western University

Scholarship@Western

8-1-2018

\title{
Magnetic resonance imaging in the diagnosis of white matter signal abnormalities.
}

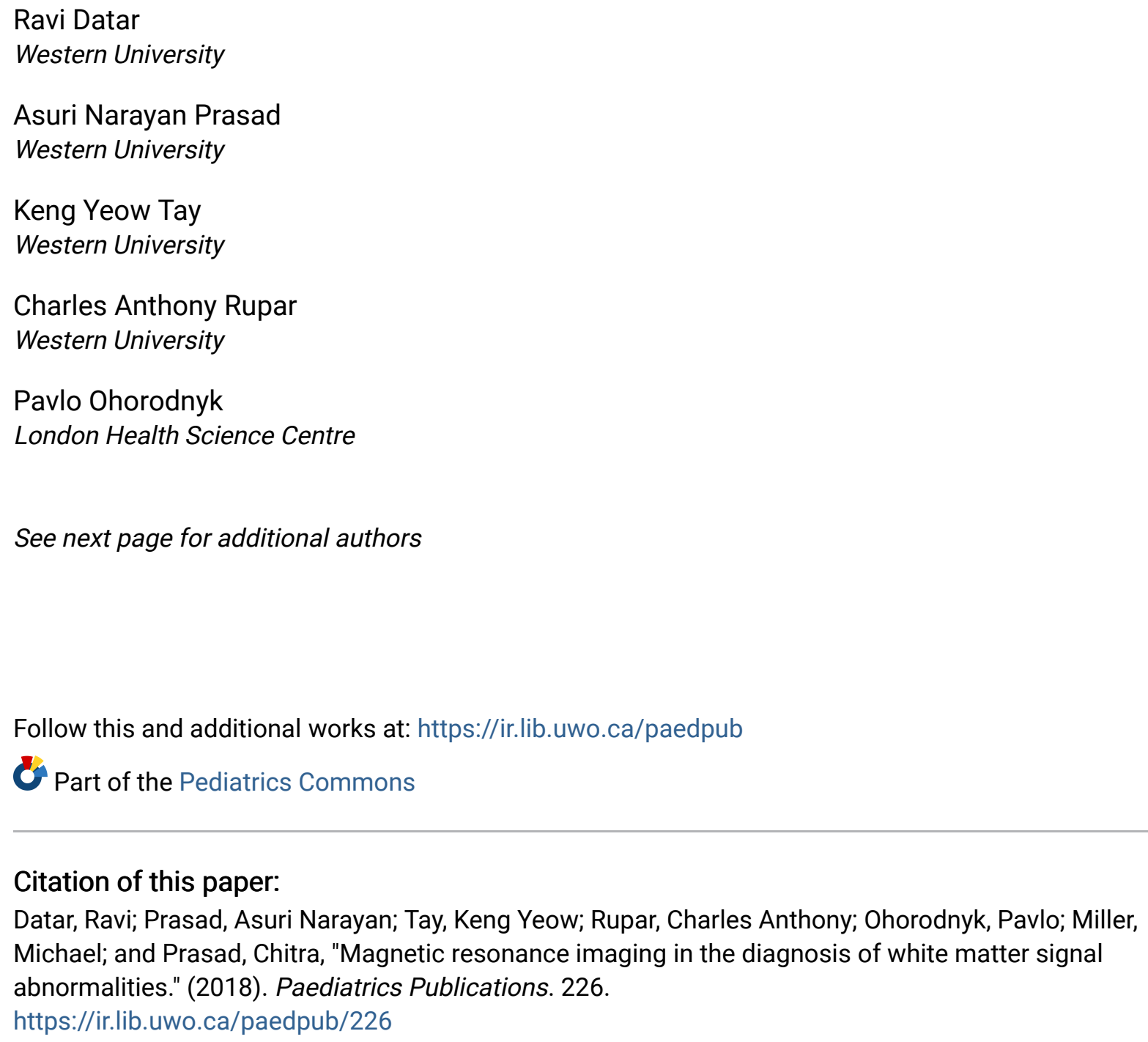

See next page for additional authors

Follow this and additional works at: https://ir.lib.uwo.ca/paedpub

Part of the Pediatrics Commons

\section{Citation of this paper:}

Datar, Ravi; Prasad, Asuri Narayan; Tay, Keng Yeow; Rupar, Charles Anthony; Ohorodnyk, Pavlo; Miller, Michael; and Prasad, Chitra, "Magnetic resonance imaging in the diagnosis of white matter signal abnormalities." (2018). Paediatrics Publications. 226.

https://ir.lib.uwo.ca/paedpub/226 


\section{Authors}

Ravi Datar, Asuri Narayan Prasad, Keng Yeow Tay, Charles Anthony Rupar, Pavlo Ohorodnyk, Michael Miller, and Chitra Prasad 


\title{
Magnetic resonance imaging in the diagnosis of white matter signal abnormalities
}

\author{
Ravi Datar ${ }^{1,2}$, Asuri Narayan Prasad ${ }^{1,3,4,5}$, Keng Yeow Tay ${ }^{1,6}$, \\ Charles Anthony Rupar ${ }^{1,3,5,7,8}$, Pavlo Ohorodnyk ${ }^{6}$, Michael Miller ${ }^{3,5}$ \\ and Chitra Prasad ${ }^{1,3,5}$
}

(3)AGE

\begin{abstract}
Background: White matter abnormalities (WMAs) pose a diagnostic challenge when trying to establish etiologic diagnoses. During childhood and adult years, genetic disorders, metabolic disorders and acquired conditions are included in differential diagnoses. To assist clinicians and radiologists, a structured algorithm using cranial magnetic resonance imaging (MRI) has been recommended to aid in establishing working diagnoses that facilitate appropriate biochemical and genetic investigations. This retrospective pilot study investigated the validity and diagnostic utility of this algorithm when applied to white matter signal abnormalities (WMSAs) reported on imaging studies of patients seen in our clinics.

Methods: The MRI algorithm was applied to 31 patients selected from patients attending the neurometabolic/neurogenetic/ metabolic/neurology clinics at a tertiary care hospital. These patients varied in age from 5 months to 79 years old, and were reported to have WMSAs on cranial MRI scans. Twenty-one patients had confirmed WMA diagnoses and 10 patients had non-specific WMA diagnoses (etiology unknown). Two radiologists, blinded to confirmed diagnoses, used clinical abstracts and the WMSAs present on patient MRI scans to classify possible WMA diagnoses utilizing the algorithm.

Results: The MRI algorithm displayed a sensitivity of $100 \%$, a specificity of $30.0 \%$ and a positive predicted value of $74.1 \%$. Cohen's kappa statistic for inter-radiologist agreement was 0.733 , suggesting "good" agreement between radiologists.

Conclusions: Although a high diagnostic utility was not observed, results suggest that this MRI algorithm has promise as a clinical tool for clinicians and radiologists. We discuss the benefits and limitations of this approach.
\end{abstract}

\section{Keywords}

White matter abnormalities, myelination, magnetic resonance imaging, diagnostic utility, diagnostic neuroradiology

\section{Introduction}

A key feature of the developing brain is myelination progression in the central nervous system, starting as early as 25 weeks into fetal development. ${ }^{1}$ Central myelinogenesis requires an intricate partnership between axons and the oligodendrocytes responsible for myelin deposition and maintenance. ${ }^{2}$ Dysfunction of either of these cell types at any of the various levels of myelin biosynthesis and turnover can lead to diseases of cranial white matter and consequential functional impairments that can lead to progressive neurological disease and eventually death. ${ }^{3}$

The clinical diagnosis of such white matter abnormalities (WMAs) continues to be of utmost importance. ${ }^{4}$ On cranial imaging studies, such as magnetic resonance imaging (MRI), WMAs can be identified by the presence of visible white matter signal abnormalities (WMSAs). A major consideration in these settings is a group of disorders called leukoencephalopathies (LEs; see Figure 1). LEs, a term applying to all brain WMAs, can be inherited or acquired. Limited
${ }^{1}$ Schulich School of Medicine and Dentistry, Western University, London, ON, Canada

${ }^{2}$ Department of Medical Genetics, London Health Sciences Centre, London, ON, Canada

${ }^{3}$ Department of Paediatrics, London Health Sciences Centre, London, ON, Canada

${ }^{4}$ Division of Clinical Neurosciences, London Health Sciences Centre, London, ON, Canada

${ }^{5}$ Children's Health Research Institute, London Health Sciences Centre, London, ON, Canada

${ }^{6}$ Department of Medical Imaging, London Health Sciences Centre, London, ON, Canada

${ }^{7}$ Department of Pathology and Laboratory Medicine, London Health Sciences Centre, London, ON, Canada

${ }^{8}$ Department of Biochemistry, London Health Sciences Centre, London, ON, Canada

\section{Corresponding author:}

Chitra Prasad, Department of Paediatrics, Section of Genetics and Metabolism, Children's Hospital, London Health Sciences Centre, 800 Commissioners Road East, London, Ontario, N6A 5W9, Canada.

Email: Chitra.Prasad@Ihsc.on.ca 


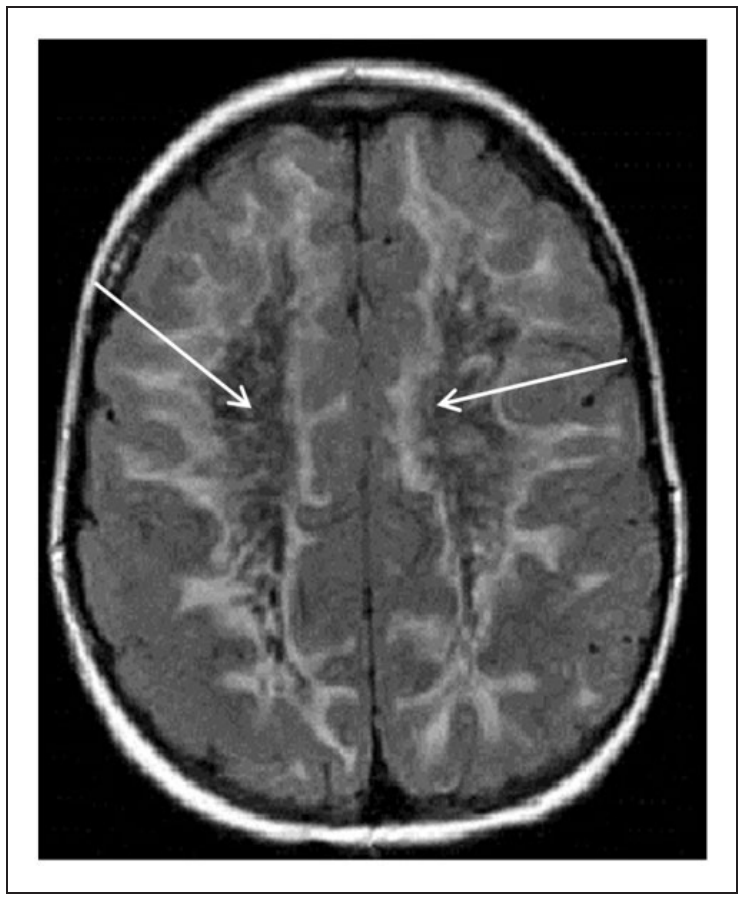

Figure 1. Axial fluid-attenuated inversion recovery magnetic resonance imaging scan of the brain showing extensive white matter hyperintensity extending to the subcortical U-fibers. The central white matter suppresses with signal similar to cerebrospinal fluid (see white arrows) in this patient with vanishing white matter disease.

treatment options add to their severity and burden of disease. ${ }^{5}$ As these disorders pose a diagnostic challenge and are time sensitive, attention has been focused on shortening the time to diagnosis and improving physician awareness. The reported prevalence of WMAs varies in the literature across different age groups and geographies. Leukodystrophies (LDs; see Figure 2) are a form of inherited LEs, and the reported prevalence of all major forms of LDs varies from $1 / 50,000$ in Germany ${ }^{6}$ to $1 / 7663$ in Utah, United States. ${ }^{7}$ However, WMAs are being recognized and reported more often in young children. ${ }^{7}$

The appropriate diagnosis of WMAs in infants and children is particularly crucial given high mortality rates for inherited progressive LDs; $85 \%$ to $90 \%$ of infants suffering from a disease such as Krabbe disease die before 2 years of age. ${ }^{8}$ The earlier the diagnosis and management of the few treatable WMAs, the better the outcome is for subsequent myelin deposition. ${ }^{9}$ Organizations such as the Global Leukodystrophy Initiative have underscored the fact that despite being members of the Society of Inherited Metabolic Disorders and the Child Neurology Society, most biochemical/clinical geneticists and pediatric neurologists find it challenging to provide differential diagnoses of some WMAs. ${ }^{10}$

A recent clinician survey suggested that only $52 \%$ of clinicians are at least moderately confident of providing a WMA diagnosis, with only $16 \%$ of clinicians being highly confident. ${ }^{10}$ Symptoms of different diseases often

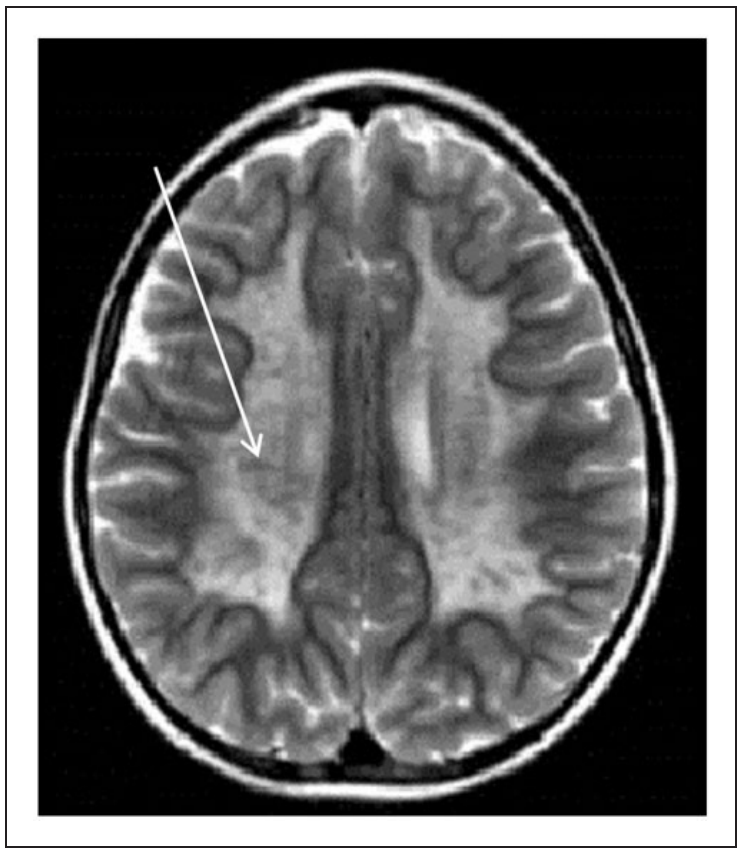

Figure 2. Axial T2-weighted magnetic resonance imaging scan of the brain showing the "tigroid" pattern (white arrow) often seen in metachromatic leukodystrophy.

overlap and present similarly in the clinic, contributing to the difficulty of establishing a differential diagnosis. ${ }^{11}$ Nonetheless, there are a few WMAs that are associated with specific clinical features that can carry diagnostic value. $^{12}$ These can be quite helpful when a clinician attempts to narrow down a differential diagnosis, such as the presence of adrenal insufficiency in patients with adrenoleukodystrophies. ${ }^{10}$

In recent years, authorities in the field of LEs have suggested that cranial MRI scans can strongly aid an accurate diagnosis of WMAs through a systematic analysis of the WMSAs present on scans. ${ }^{10,12}$ A protocol has been proposed that uses cranial MRI analysis as a keystone in the diagnostic algorithm to expedite the diagnosis of WMAs. ${ }^{10,12}$ Different imaging sequences, such as T1-weighted (T1W), T2-weighted (T2W) and fluid-attenuated inversion recovery (FLAIR) signals vary in their sensitivity to different structural components in brain matter, which can be analyzed to create characteristic patterns for certain categories of WMAs. The diagnostic algorithm provides clinicians with a step-by-step approach to narrowing down a patient's diagnosis to a group of diseases that exhibit WMSA similarities on MRI scans. ${ }^{10,12}$ This algorithm takes into consideration the type of myelin pathology (hypomyelination, demyelination, delayed myelination and dysmyelination) as well as the location, symmetry, regional distribution, signal intensity and change over time of the WMSAs to categorize certain WMAs. ${ }^{10}$ Authors of this approach have statistically validated the categorization of WMAs based on WMSA patterns in their clinics. ${ }^{13}$ Through the application of the algorithm, recognition of WMSA patterns can lead to a 
group of differential diagnoses after consideration of the patient's clinical data. A clinician can then order a focused set of molecular genetic testing for these diagnostic shortlists, rather than subjecting the patient to a larger set of tests that can be expensive, demanding on the patient and sometimes inconclusive. ${ }^{12,14}$

At the London Health Sciences Centre in London, Ontario, Canada, approximately 270 patients of varying ages have been diagnosed with WMAs via confirmative genetic/biochemical testing since 2004 . The primary objective of this study is to evaluate the utility of such a diagnostic system, with the goal of raising physician awareness and implications for wider clinical use. It is worth investigating this MRI-centric algorithm for three main reasons: (a) the current lack of a systematic diagnostic approach to WMSAs, (b) the high mortality rate and short life expectancies associated with some WMAs and (c) the potential to improve the accuracy and shorten time to WMA diagnosis. ${ }^{15}$ This project aims to study the utility of the algorithm in deducing WMA diagnoses as well as its reliability between multiple algorithm users. We hypothesized that this MRI algorithm would display diagnostic utility as a test with both high accuracy and inter-rater reliability.

\section{Methods}

\section{Participant selection criteria}

A total of 270 patients in the neurometabolic/neurogenetic/metabolic/neurology clinics were sequentially assessed for inclusion. The patients included in this study were referred to these clinics at the London Health Sciences Centre in London, Ontario, Canada, between 2004 and 2015. These patients completed a consultation and at least one cranial MRI scan. The MRI scans for these patients were confirmed by a neurologist as displaying WMSAs.

In the selection process, some patients were excluded on the basis that their WMSAs were not attributed primarily to WMAs, but rather to other factors such as acute trauma. After applying the above-mentioned selection criteria, 31 patients (14 males, 17 females) were selected for this study. The ages of the patients ranged from five months to 79 years. Twenty-one patients had a specific diagnosis for their WMAs based on confirmative molecular and biochemical testing, and 10 had non-specific WMAs (i.e. non-confirmative based on clinical assessment supported by negative molecular/biochemical test results). For purposes of patient confidentiality, all patients were anonymized and given a numerical code. Identifiable patient information and corresponding numerical codes were stored on a virtual password-protected document, which was accessible only by the principal investigator (CP) and student researcher (RD). Patients' dates of birth were the only personal information disclosed to the entire research team. This study was approved by the
Health Sciences Research Ethics Board at Western University, London, Ontario, Canada.

\section{Diagnostic algorithm}

The first phase of this study involved an independent analysis of all cranial MRI scans by two radiologists, yielding two sets of data for each patient. For each scan, the radiologists were given the dates when scans were performed since myelination progress is age dependent.

The radiologists included in this study, KYT and PO, had 11 years and one year of experience respectively at the time of MRI scan interpretation. After analysis of T1W, T2W and FLAIR signals, the radiologists deduced the type of myelin pathology as shown in the scans (see Figure 3; readers interested in reviewing more images are invited to reference Schiffman and van der Knaap, 2009 ${ }^{14}$ ). The first point of determination was establishing the presence/absence of hypomyelination. If the radiologists observed a hypomyelination pattern present on the scans, they proceeded to look at WMSAs in the cerebellum, the basal ganglia and the presence/absence of global atrophy to determine the subtype of hypomyelination present. After determining this, the radiologists arrived at a category of WMAs. In case there was no hypomyelination pattern present, the radiologists analyzed the MRI scan for the location, symmetry and regional distribution/isolation of the WMSAs. This was performed to confirm the presence of dysmyelination or demyelination and to arrive at a specific group of WMAs with shared characteristics.

Once the radiologists had arrived at a particular group of conditions, they were given abstracted clinical data including both neurological and extra-neurological symptoms. Using this clinical information, the radiologists were asked to reduce the group of conditions in a particular category to one to three differential diagnoses of the patient's WMA. With each differential diagnosis, the radiologists were asked to associate a level of confidence from 1 to 10 with 1 being not confident and 10 being very confident. A confidence value of 4 was selected as the threshold; in a clinical scenario, a differential diagnosis with such a low likelihood is unlikely to be considered. Thus, any differential diagnosis with a confidence value $\leq 4$ was discarded. If the WMSAs were unable to fit into the algorithm and the radiologists were unable to reach a category, a nonspecific diagnosis was recorded.

The WMSAs in the MRI scans were either diagnostic of a specific genetic/acquired condition (molecular/ biochemical testing was positive) or non-specific (molecular/biochemical testing was negative). For all patients, the radiologists were blinded to the confirmed diagnosis, or "correct answer." Based on whether the algorithm correctly deduced confirmed specific diagnoses or non-specific diagnosis when molecular/ biochemical tests were actually positive, values for true-positive and false-negative (respectively) outcomes 


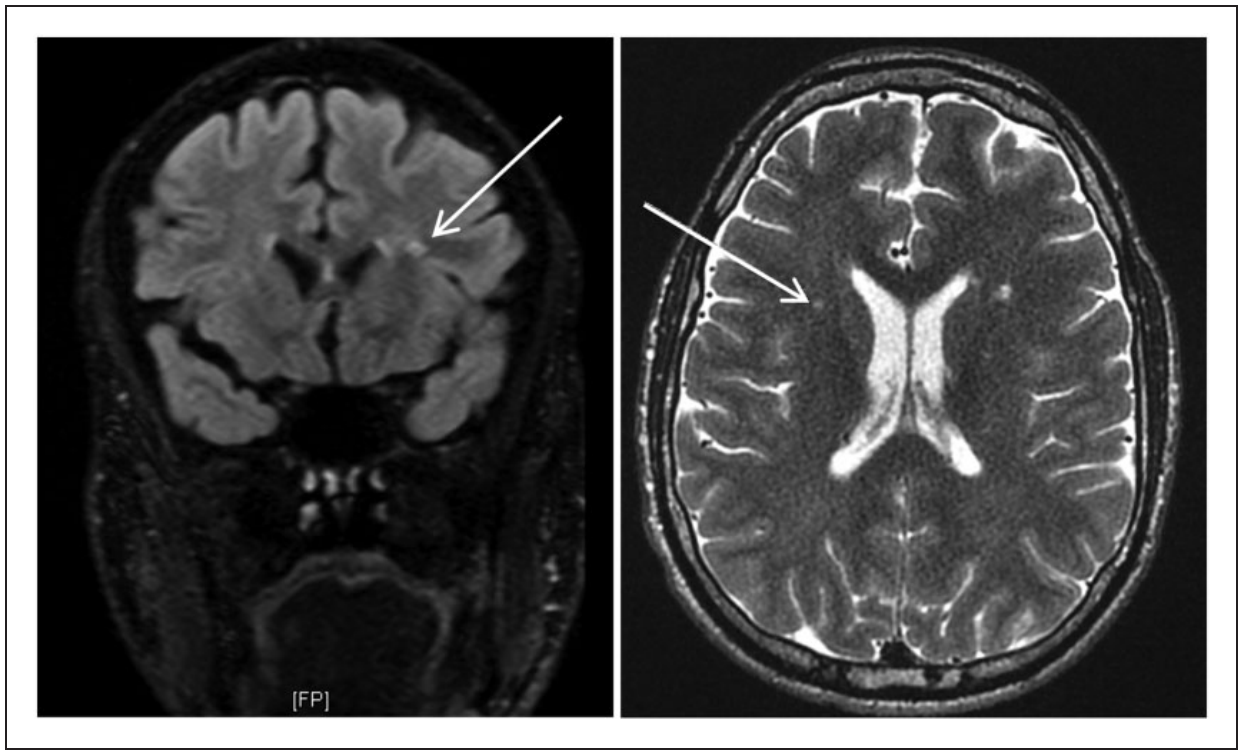

Figure 3. Fluid-attenuated inversion recovery (left) and T2-weighted (right) magnetic resonance imaging scans for patient 013 . Using the algorithm, both radiologists confirmed a non-hypomyelination pattern. Multifocal white matter signal abnormalities (WMSAs) were noted (see white arrows for a focus). These WMSAs progressed to confluence over seven years of longitudinal scans. After progressing through the algorithm, both radiologists used this information to arrive at category 7.17 (true positive outcome). This category correctly included the confirmed differential diagnosis of Fabry disease.

were recorded. On the other hand, if the algorithm results were inconclusive of a diagnosis for confirmed non-specific WMAs or suggested a diagnosis when molecular/biochemical tests were actually negative, values for true-negative or false-positive (respectively) outcomes were recorded. All statistical analyses were performed using GraphPad Prism ${ }^{\circledR} 6$ software. The positive/negative testing outcomes allowed for sensitivity, specificity and positive predictive value (PPV) statistics, which were used to determine the utility of the algorithm in diagnosing WMAs. Cohen's kappa statistic was used to quantify algorithm consistency in terms of inter-rater agreement between the radiologists' outcomes.

\section{Results}

Data collection was performed over the span of seven months at the radiology department at Children's Hospital in London, Ontario, Canada. Both radiologists expressed concerns with isolating one to three differential diagnoses from the specified category. Since the professional responsibilities of radiologists do not include diagnosing a patient, but rather solely identifying patterns in an MRI scan, their evaluation of differential diagnoses was not considered an accurate representation of the algorithm's validity. Thus, all test outcomes were based on whether the algorithm allowed the radiologists to arrive at the correct category rather than the correct differential diagnoses within the category.

Although this study began with 31 patients, patient 018 was excluded from this study after data collection (see Table 1). Both radiologists felt that the algorithm was not applicable for this patient. They were unable to deduce hypomyelination, as there was only one set of images available for the patient rather than sequential scans at least six months apart. With the exclusion of this patient, the final sample consisted of 30 patients, and the prevalence of specific confirmed diagnoses was $66.7 \%$.

Table 2 summarizes these diagnostic outcomes and whether each positive/negative conclusion was correct (true) or incorrect (false).

The diagnostic utility of this clinical diagnostic algorithm was quantified through four statistics: sensitivity, specificity, the PPV and Cohen's kappa statistic. Sensitivity was used as a measure of the algorithm to correctly identify patients with a specific diagnosis. ${ }^{16}$ Specificity was used to correctly identify patients with a non-specific diagnosis. ${ }^{16}$ The PPV was used to quantify the proportion of all specific diagnoses produced by the algorithm in which the suggested category correctly contained the confirmed diagnosis. ${ }^{16}$ Cohen's kappa statistic was used to determine inter-rater reliability.

Sensitivity, specificity and PPV values are summarized in Table 3. Both radiologists successfully recognized a specific diagnosis for all specifically diagnosed patients (i.e. recorded no false-negatives). Hence, the individual and mean sensitivity and negative predictive value (NPV) of the algorithm was $100 \%$ for this patient sample. The ability of the algorithm to identify non-specific diagnoses as non-specific via the algorithm, however, was much lower at a mean specificity of $30.0 \%$. The mean PPV was calculated as $74.1 \%$, implying that for every four patients for whom a category was reached, approximately three of these patients had a confirmed specific diagnosis. Out of all 
Table 1. Summary of diagnostic outcomes after the analysis of 31 patients with WMAs.

\begin{tabular}{|c|c|c|c|}
\hline Patient ID & Confirmed diagnosis & $\begin{array}{l}\text { Radiologist } 1 \\
\text { outcome }\end{array}$ & $\begin{array}{l}\text { Radiologist } 2 \\
\text { outcome }\end{array}$ \\
\hline 001 & MELAS & TP & TP \\
\hline 002 & Vanishing white matter disease & TP & TP \\
\hline 003 & Phenylketonuria & TP & TP \\
\hline 004 & Adrenomyeloneuropathy with adrenal insufficiency & TP & TP \\
\hline 005 & Multiple sulfatase deficiency & TP & TP \\
\hline 006 & Leigh syndrome & TP & TP \\
\hline 007 & Fabry disease & TP & TP \\
\hline 008 & Phenylketonuria & TP & TP \\
\hline 009 & Neuronal ceroid lipofuscinosis & TP & TP \\
\hline 010 & MELAS & TP & TP \\
\hline 011 & 5MTHFR deficiency & TP & TP \\
\hline 012 & X-linked adrenoleukodystrophy & TP & TP \\
\hline 013 & Fabry disease & TP & TP \\
\hline 014 & Metachromatic leukodystrophy & TP & TP \\
\hline 015 & MELAS & TP & TP \\
\hline 016 & CADASIL & TP & TP \\
\hline 017 & NARP-like mitochondrial disorder & TP & TP \\
\hline $018^{\mathrm{a}}$ & Coffin-Siris syndrome & TP & FN \\
\hline 019 & Krabbe disease & TP & TP \\
\hline 020 & 48, XXXY syndrome & TP & TP \\
\hline 021 & GMI gangliosidosis & TP & TP \\
\hline 022 & NON-SPECIFIC & $\mathrm{FP}$ & FP \\
\hline 023 & NON-SPECIFIC & TN & FP \\
\hline 024 & NON-SPECIFIC & FP & FP \\
\hline 025 & NON-SPECIFIC & FP & FP \\
\hline 026 & NON-SPECIFIC & TN & TN \\
\hline 027 & NON-SPECIFIC & TN & FP \\
\hline 028 & NON-SPECIFIC & TN & FP \\
\hline 029 & NON-SPECIFIC & $\mathrm{FP}$ & FP \\
\hline 030 & NON-SPECIFIC & TN & FP \\
\hline 031 & NON-SPECIFIC & FP & FP \\
\hline
\end{tabular}

Patients 001-021 have been diagnosed with a specific WMA and patients 022-031 have been non-specifically diagnosed (etiology unknown). Values shown include true positive (TP), true negative (TN), false positive (FP) and false negative (FN). Outcomes are presented by individual radiologist. ${ }^{\mathrm{a}}$ Patient 018 was excluded from the study and subsequent statistical analysis, thus altering the prevalence of a specific diagnosed condition to $66.7 \%$. CADASIL: cerebral autosomal dominant arteriopathy with subcortical infarcts and leukoencephalopathy; ID: identification; MELAS: mitochondrial myopathy, encephalopathy, lactic acidosis, stroke-like episodes; WMAs: white matter abnormalities.

Table 2. Summary of diagnostic outcomes after the analysis of 30 patients by two radiologists using the MRI algorithm.

\begin{tabular}{lcccc}
\hline & TP & FP & TN & FN \\
\hline Radiologist 1 & 20 & 5 & 5 & 0 \\
Radiologist 2 & 20 & 9 & 1 & 0 \\
TOTAL & 40 & 14 & 6 & 0 \\
\hline
\end{tabular}

Twenty patients were diagnosed with a specific WMA and 10 were given non-specific diagnoses (etiology unknown). Prevalence of a specific diagnosed condition was $66.7 \%$. Values shown include true positive (TP), true negative (TN), false positive (FP) and false negative (FN). Outcomes are presented by individual radiologist and as combined totals. MRI: magnetic resonance imaging; WMA: white matter abnormality. the true-positive outcomes produced by the radiologists, they did not always reach a category that contained the correct differential diagnosis. For all truepositives, an accurate category was reached on average $62.5 \%$ of the time (Radiologist $1-75 \%$ and Radiologist $2-50 \%)$. The calculated Cohen's kappa was 0.733 (see Table 4), suggesting good agreement between the radiologists. ${ }^{17}$

\section{Discussion}

It was originally hypothesized that the MRI algorithm would yield diagnostic utility as a test with high 
Table 3. Summary of diagnostic statistics after the analysis of 30 patients by two radiologists using the MRI algorithm. Sensitivity $=T P /(T P+F N)$; Specificity $=T N /(T N+F P) ; P P V=T P /(T P+F P)$; $\mathrm{NPV}=\mathrm{TN} /(\mathrm{TN}+\mathrm{FP})$. Point estimates are followed by $95 \%$ confidence intervals in brackets.

\begin{tabular}{lllll}
\hline & Sensitivity & Specificity & PPV & NPV \\
\hline Radiologist 1 & $100(-)$ & $50.0(19.0-81.0)$ & $80.0(64.3-95.7)$ & $100(-)$ \\
Radiologist 2 & $100(-)$ & $10.0(-8.59-28.6)$ & $69.0(52.1-85.8)$ & $100(-)$ \\
MEAN & $100(-)$ & $30.0(1.60-58.4)$ & $74.1(57.5-90.6)$ & $100(-)$ \\
\hline
\end{tabular}

Twenty patients were diagnosed with a specific WMA and 10 were given non-specific diagnoses (etiology unknown). Prevalence of a specific diagnosed condition was $66.7 \%$. Values shown include true positive (TP), true negative (TN), false positive (FP) and false negative (FN). Outcomes are presented by individual radiologist and as combined totals. MRI: magnetic resonance imaging; NPV: negative predictive value; PPV: positive predictive value; WMA: white matter abnormality.

Table 4. Summary of paired outcomes provided by both radiologists on a patient-by-patient basis. The top row refers to the diagnostic outcome (TP/FP/TN/FN) from the first radiologist. The left column refers to the diagnostic outcome (TP/FP/TN/FN) from the second radiologist. From this tabulation, the observed kappa statistic was calculated via GraphPad Prism ${ }^{\circledR} 6$ software as 0.733 .

\begin{tabular}{llllll}
\hline & \multicolumn{4}{l}{ Radiologist 1} \\
\cline { 3 - 5 } & & TP & FP & TN & FN \\
\hline Radiologist 2 & TP & 20 & 0 & 0 & 0 \\
& FP & 0 & 5 & 4 & 0 \\
& TN & 0 & 0 & 1 & 0 \\
& FN & 0 & 0 & 0 & 0 \\
\hline
\end{tabular}

Twenty patients were diagnosed with a specific WMA and 10 were given non-specific diagnoses (etiology unknown). Prevalence of specific diagnosed condition was $66.7 \%$. Shaded boxes indicate instances where both radiologists were in agreement. FN: false negative; FP: false positive; MRI: magnetic resonance imaging; TN: true negative; TP: true positive; WMA: white matter abnormality.

accuracy and inter-rater reliability. The perfect sensitivity and NPVs of this algorithm can be attributed to the lack of any false-negatives diagnostic outcomes. For patients with a specific confirmed diagnosis, the radiologists always arrived at a category and, thus, produced a true-positive outcome. A perfect sensitivity and low specificity have the implication of producing occasional false-positives, which was seen in this group of diagnostic outcomes. Sensitivity takes into consideration only whether a category is reached, but not whether the category is the one containing the correct diagnosis. The latter is a better measure of utility for clinicians, as reaching a working category that omits the correct differential diagnosis is of no benefit. For all true positives, a correct differential diagnosis-containing category was reached $62.5 \%$ of the time. This statistic combined with the lower specificity and PPV values downplay the utility of the algorithm. The low specificity suggests that specific outcomes were overproduced, i.e. even those WMSAs that were due to nonspecific WMAs were incorrectly categorized into specific categories. This can be considered a limitation of the study and the utility of the algorithm may be dependent on the individuals who are using it, the nature of imaging findings and the timing/availability of sequential scans. Certain patients, however, displayed WMSAs that were characteristic enough to fit into the algorithm. The nature of these WMSAs to manifest similarly to WMSAs of a specific diagnosis contributed to the algorithm's lower responsiveness to non-specific WMAs.

Specificity and PPV values varied substantially between the two radiologists $(50.0 \%$ vs. $10.0 \%$ and $80.0 \%$ vs. $69.0 \%$, respectively). A Cohen's kappa statistic of 0.733 suggests a "good" level of agreement between the two radiologists. Considering that there were only two raters in this study, however, it is uncertain whether these values would change if a larger group of radiologists were consulted. The radiologists also varied in their levels of training; one consultant was an attending neuroradiologist with many years of diagnostic experience and the other consultant was a recent neuroradiology fellow. This difference in experience may have also contributed to a difference in diagnostic outcomes. It would be prudent to include $>2$ radiologists in future investigations for a more representative assessment of the algorithm's inter-rater reliability, accuracy and to produce narrower confidence intervals.

A decision tree representation of the algorithm is shown (Figure 4) in which the first decision to be made is whether hypomyelination or another myelin pathology is present. Hypomyelination is a term reserved for WMAs that have a significant and permanent deficit in the deposition of myelin. ${ }^{9}$ To confirm the permanent nature of the myelin deficit, MRI scans must be viewed longitudinally. Hypomyelination requires multiple sequential MRI scans that are a minimum of six months apart. ${ }^{9}$ Without these sequential scans, there are insufficient data to distinguish between hypomyelination and delayed myelination, the latter of which is not permanent and improves over time. ${ }^{10}$ This multiple sequential imaging requirement should be emphasized in future investigations.

During data collection, it was noted that the algorithm was not able to distinguish WMAs that had been well managed early on in life. Phenylketonuria is a disorder that can be detected in newborn screening, and 


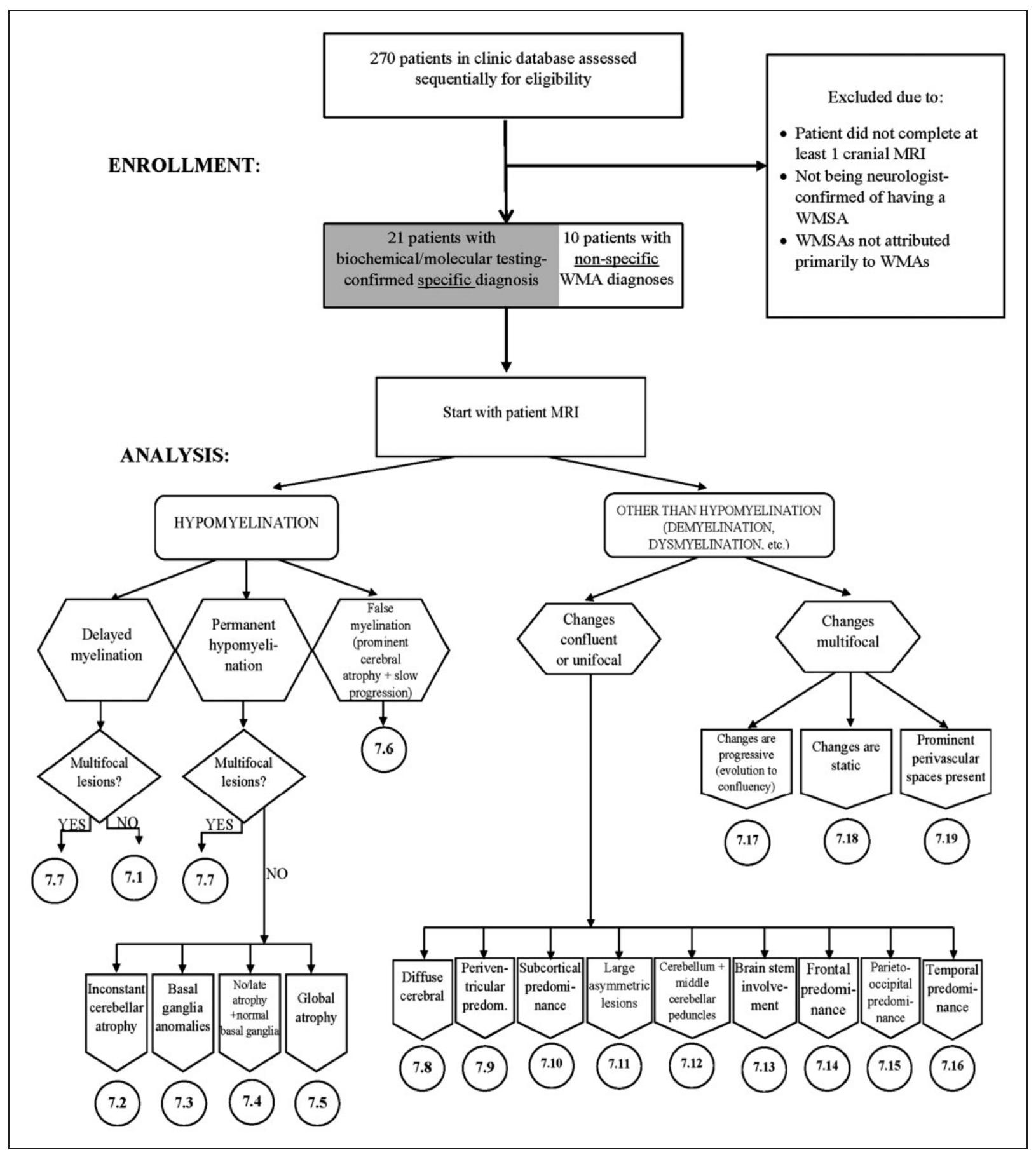

Figure 4. The magnetic resonance imaging (MRI) algorithm used for the analysis of white matter signal abnormalities (WMSAs) on patient cranial scans. The radiologist began by identifying whether the signal abnormality was indicative of hypomyelination or another type of myelin pathology (dysmyelination, demyelination, etc.). Within the type of myelination present, the radiologist proceeded through the algorithm down the paths that were most applicable to the WMSAs on the scans. Once at a concluding category, denoted by 7 .\#\#, the radiologist consulted the patient clinical data to determine differential diagnoses contained in that numerical category. Up to three possible differential diagnoses were allowed for each patient. For each differential diagnosis, a confidence value of 1-10 was assigned. This algorithm, the categorical organization and legend abbreviations are identical to those published by Parikh et al., but the presentation is different. ${ }^{10}$

the consistent following of a low-phenylalanine diet can result in a very normal phenotype. Similarly, Fabry disease can have variable neurological presentations. Individuals with well-managed diseases can have diminished severity of abnormalities seen on MRI scans. The algorithm demands for distinct, distinguishable WMSA patterns, and the absence of such patterns, makes it difficult for the algorithm to sense well-managed WMAs.

The algorithm also displayed difficulties with endstage WMAs. After a certain amount of time and disease progression, WMSAs become so severe on MRI scans that the originally distinguishable patterns 


\begin{tabular}{|c|c|c|c|c|c|c|c|c|c|}
\hline \multicolumn{10}{|l|}{ LEGEND: } \\
\hline \multicolumn{10}{|c|}{$\begin{array}{l}\text { LEGEND: } \\
\text { 4H: hypomyelination, hypodontia and hypogonadotropic hypogonadism }\end{array}$} \\
\hline \multicolumn{6}{|c|}{ ADLD: autosomal dominant leukodystrophy with autonomic symptoms } & \multicolumn{4}{|c|}{ HEMS: hypomyelination of early myelinating structures } \\
\hline \multicolumn{6}{|c|}{ APBD: adult polyglucosan body disease } & \multicolumn{4}{|c|}{ HIV: human immunodeficiency virus } \\
\hline \multicolumn{6}{|c|}{ BCAA: branched-chain amino acid } & \multicolumn{4}{|c|}{ LBSL: leukoencephalopathy with brai } \\
\hline \multicolumn{6}{|c|}{$\begin{array}{l}\text { CADASIL: cerebral autosomal dominant arteriopathy with subcortical infarcts and } \\
\text { leukoencephalopathy }\end{array}$} & \multicolumn{4}{|c|}{$\begin{array}{l}\text { elevation } \\
\text { LD: leukodystrophy }\end{array}$} \\
\hline \multirow{2}{*}{\multicolumn{6}{|c|}{$\begin{array}{l}\text { CARASIL: cerebral autosomal recessive arteriopathy with subcortical infarcts and } \\
\text { leukoencephalopathy }\end{array}$}} & \multicolumn{4}{|c|}{ LE: leukoencephalopathy } \\
\hline & & & & & & \multirow{2}{*}{\multicolumn{4}{|c|}{$\begin{array}{l}\text { LTBL: leukoencephalopathy with thalamus and brainstem involvement and high } \\
\text { lactate }\end{array}$}} \\
\hline \multicolumn{6}{|c|}{ CMV: cytomegalovirus } & & & & \\
\hline \multicolumn{6}{|c|}{ CRMCC: cerebroretinal microangiopathy with calcifications and cysts } & \multicolumn{4}{|c|}{ MLC: megalencephalic leukoencephalopathy with subcortical cysts } \\
\hline \multicolumn{6}{|c|}{ CTX: cerebrotendinous xanthomatosis } & \multicolumn{4}{|c|}{ MPS: mucopolysaccharidoses } \\
\hline \multicolumn{6}{|c|}{ DRPLA: dentatorubral-pallidoluysian atrophy } & \multicolumn{4}{|c|}{ MSUD: maple syrup urine disease } \\
\hline \multicolumn{6}{|c|}{$\begin{array}{l}\text { EIF2B-related disorders: vanishing white matter disease or childhood ataxia with } \\
\text { diffuse CNS hypomyelination) }\end{array}$} & \multicolumn{4}{|c|}{ NCL: neuronal ceroid lipofuscinosis } \\
\hline \multirow{2}{*}{\multicolumn{6}{|c|}{ HABC: hypomyelination with atrophy of the basal ganglia and cerebellum }} & \multirow{2}{*}{\multicolumn{4}{|c|}{ PKU: phenylketonuria }} \\
\hline & & & & & & & & & \\
\hline \multicolumn{6}{|c|}{ HCC: hypomyelination with congenital cataract } & \multicolumn{4}{|c|}{$\begin{array}{l}\text { PMLD: Pelizaeus-Merzbacher like-disease } \\
\text { X-ALD: X-linked adrenoleukodystrophy }\end{array}$} \\
\hline 7.1 & 7.2 & & & 7.3 & & 7.4 & 7.5 & & 7.6 \\
\hline $\begin{array}{l}\rightarrow \text { SOX10 related } \\
\text { disorders } \\
\rightarrow \text { MCT8 related } \\
\text { disorders } \\
\rightarrow \text { Other neuronal } \\
\text { disorders }\end{array}$ & $\begin{array}{l}\rightarrow 4 \mathrm{H} \text { syndrome } \\
\rightarrow \text { HABC } \\
\rightarrow \text { ODDD } \\
\rightarrow \text { Salla disease } \\
\rightarrow \text { Cockayne syn }\end{array}$ & drome & $\begin{array}{l}\rightarrow \text { Fuc } \\
\rightarrow \text { HA } \\
\rightarrow \text { OD } \\
\rightarrow \text { Mu } \\
\text { type I }\end{array}$ & $\begin{array}{l}\text { sidosis } \\
\text { C } \\
\text { D } \\
\text { lipidosis }\end{array}$ & & $\begin{array}{l}\text { l minus syndrome } \\
\text { C (N.B. some region } \\
\text { ave low T1 signal } \\
\text { MS } \\
\text { ID } \\
\text { ILD } \\
\text { la disease } \\
\text { X10-associated } \\
\text { lers }\end{array}$ & $\begin{array}{l}\rightarrow \text { Infantile Sialic } \\
\text { Acid Storage } \\
\text { Disease } \\
\rightarrow \text { Aicardi- } \\
\text { Goutieres } \\
\text { syndrome }\end{array}$ & $\begin{array}{l}\rightarrow \text { Serine synthesis def } \\
\rightarrow \text { NCL } \\
\rightarrow \text { Early onset GM1 an } \\
\rightarrow \text { Mitochondrial disor } \\
\rightarrow \text { Fumarate Hydratase } \\
\rightarrow \text { Band-like intracrani } \\
\text { gyration and polymicr } \\
\rightarrow \text { Neuropathic form o } \\
\text { osteopetrosis } \\
\rightarrow \text { AGC1-rel disorders } \\
\rightarrow \text { HSPD1-rel disorder } \\
\rightarrow \text { AIMP1-rel disorder } \\
\rightarrow \text { GPR56-rel disorder }\end{array}$ & $\begin{array}{l}\text { ects } \\
\text { d GM2 } \\
\text { lers } \\
\text { deficiency } \\
\text { al calcification with sim. } \\
\text { glia } \\
\text { malignant infantile }\end{array}$ \\
\hline 7.7 & \multicolumn{2}{|l|}{ 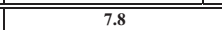 } & \multicolumn{4}{|c|}{$\begin{array}{lll}7.9 & \end{array}$} & 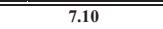 & 7.11 & 7.12 \\
\hline $\begin{array}{l}\rightarrow 18 \mathrm{q} \text { minus } \\
\text { syndrome } \\
\rightarrow \text { HCC } \\
\rightarrow \text { Galactosemia } \\
\text { type I } \\
\rightarrow \text { Adenylosuccinate } \\
\text { lyase deficiency } \\
\rightarrow \text { Aspartylglucos- } \\
\text { aminuria } \\
\rightarrow \text { GPR56 related } \\
\text { disorders } \\
\rightarrow \text { Dystrogly- } \\
\text { canopathies } \\
\rightarrow \text { D-2- } \\
\text { hydroxyglutaric } \\
\text { aciduria }\end{array}$ & \multicolumn{2}{|c|}{$\begin{array}{l}\rightarrow \text { MLC } \\
\rightarrow \text { elF2B related disorder } \\
\rightarrow \text { Laminin alpha-2 } \\
\text { deficiency } \\
\rightarrow \text { Some mitochondrial } \\
\text { defects } \\
\rightarrow \text { Inborn errors of } \\
\text { metabolism (including } \\
\text { Molybdenum cofactor } \\
\text { deficiency, glutaric aciduria } \\
\text { II, dihydropterine reductase } \\
\text { deficiency, BCAA } \\
\text { disorders, homocystinuria) } \\
\rightarrow \text { early onset peroxisomal } \\
\text { disorders } \\
\rightarrow \text { end state of all } \\
\text { progressive WM disease }\end{array}$} & \multicolumn{4}{|c|}{$\begin{array}{l}\rightarrow \text { metachromatic LD } \\
\rightarrow \text { Krabbe disease } \\
\rightarrow \text { LBSL } \\
\rightarrow \text { Spares arcuate fibers } \\
\rightarrow \text { Sjogren Larsson syndrome } \\
\rightarrow \text { APBD } \\
\rightarrow \text { ODDD } \\
\rightarrow \text { Inborn errors of metabolism (PKU, } \\
\text { FA2H-related disorders, adenylosiccuinate } \\
\text { lyase deficiency, glutaric aciduria type II, } \\
\text { mannosidosis) } \\
\rightarrow \text { Later onset neurodegenerative disorders } \\
\text { (e.g. NCL, Nieman Pick C (NB often early } \\
\text { cerebral atrophy)) } \\
\rightarrow \text { acquired disorders like periventricular } \\
\text { leuomalacia and HIV-related } \\
\text { encephalopathy }\end{array}$} & $\begin{array}{l}\rightarrow \text { L2- } \\
\text { hypoxyglutaric } \\
\text { aciduria } \\
\rightarrow \text { Canavan disease } \\
\rightarrow \text { Keams-Sayre } \\
\text { syndrome } \\
\rightarrow \text { Propionic } \\
\text { academia } \\
\rightarrow \text { Urea cycle defects } \\
\rightarrow \text { Ribose-5- } \\
\text { phosphate isomerase } \\
\text { deficiency } \\
\rightarrow \text { LTBL }\end{array}$ & $\begin{array}{l}\rightarrow \text { HDLS } \\
\rightarrow \text { L2-hydroxyglutaric } \\
\text { aciduria } \\
\rightarrow \text { CRMCC } \\
\rightarrow \text { Mitochondrial } \\
\text { diseases } \\
\rightarrow \text { Most infectious and } \\
\text { inflammatory } \\
\text { disorders (acquired) } \\
\rightarrow \text { Inborn errors of } \\
\text { metabolism such as } \\
\text { urea cycle disorders }\end{array}$ & $\begin{array}{l}\rightarrow \text { CTX } \\
\rightarrow \text { Peroxisomal } \\
\text { disorders } \\
\rightarrow \text { Alexander Disease } \\
\rightarrow \text { LBSL } \\
\rightarrow \text { ADLD } \\
\rightarrow \text { Histiocytosis } \\
\rightarrow \text { Early onset MSUD } \\
\rightarrow \text { Premutation fragile } \\
\text { X syndrome } \\
\rightarrow \text { Heroin and cocaine } \\
\text { toxicity } \\
\rightarrow \text { FA2H related } \\
\text { disorders (atrophy) } \\
\rightarrow \text { Mitochondrial LEs }\end{array}$ \\
\hline 7.13 & 7.14 & 7.15 & & & & & 7.17 & 7.18 & 7.19 \\
\hline $\begin{array}{l}\rightarrow \rightarrow \text { LBSL } \\
\rightarrow \text { LTBL } \\
\rightarrow \text { HSBL } \\
\rightarrow \text { ADLD } \\
\rightarrow \text { Peroxisomal } \\
\text { disorders } \\
\rightarrow \text { APBD } \\
\rightarrow \text { Wilson } \\
\text { disease } \\
\rightarrow \text { Alexander } \\
\text { disease } \\
\rightarrow \text { Leigh } \\
\text { syndrome } \\
\rightarrow \text { DRPLA } \\
\rightarrow \text { Mitochondrial } \\
\text { LEs } \\
\end{array}$ & $\begin{array}{l}\rightarrow \text { Alexander } \\
\text { disease } \\
\rightarrow \text { Metachromatic } \\
\text { LD } \\
\rightarrow \text { Frontal variant } \\
\text { of X-ALD } \\
\rightarrow \text { HDLS } \\
\rightarrow \text { Aicardi- } \\
\text { Goutieres } \\
\text { Syndrome } \\
\rightarrow \text { Laminin alpha- } \\
2 \text { deficiency }\end{array}$ & $\begin{array}{l}\rightarrow \text { Krabbe } \\
\text { disease } \\
\rightarrow \text { X-ALD } \\
\rightarrow \text { Early o } \\
\text { peroxison } \\
\text { disorders } \\
\rightarrow \text { Neonat } \\
\text { hypoglyce } \\
\rightarrow \text { APBD }\end{array}$ & & $\begin{array}{l}\rightarrow \text { Menl } \\
\text { disease } \\
\rightarrow \text { Herp } \\
\text { simplex } \\
\text { enceph } \\
\rightarrow \text { Aicar } \\
\text { Goutier } \\
\text { syndron } \\
\rightarrow \text { Cong } \\
\text { CMV } \\
\rightarrow \text { RNA } \\
\text { deficier }\end{array}$ & & $\begin{array}{l}\rightarrow \text { HDLS } \\
\rightarrow \text { APBD } \\
\rightarrow \text { L2-hydroxyglut } \\
\rightarrow \text { LBSL } \\
\rightarrow \text { HBSL } \\
\rightarrow \text { Urea cycle disol } \\
\rightarrow \text { HMG-CoA lyas } \\
\rightarrow \text { Histiocytosis } \\
\rightarrow \text { Incontenitia pig } \\
\rightarrow \text { Vasculopathies } \\
\text { Fabry, Susac synd } \\
\text { vasculitis) } \\
\rightarrow \text { Multiple scleros } \\
\text { acute disseminated } \\
\rightarrow \text { progressive mul } \\
\rightarrow \text { mitochondrial d } \\
\rightarrow \text { Subacute Sclero }\end{array}$ & $\begin{array}{l}\text { aric aciduria } \\
\text { ders } \\
\text { e deficiency } \\
\text { menti } \\
\text { (CADASIL, CARASIL } \\
\text { rome, arteriolosclerosis } \\
\text { is, neuromyelitic optica } \\
\text { lencephalomyelitis } \\
\text { tifocal LE } \\
\text { iseases } \\
\text { sing Panencephalitis }\end{array}$ & $\begin{array}{l}\rightarrow 18 \mathrm{q} \text { minus } \\
\text { syndrome } \\
\rightarrow \text { Sjogren Larsson } \\
\text { syndrome } \\
\rightarrow \text { RNAse T2 defici } \\
\text { LE } \\
\rightarrow \text { Congenital CMV }\end{array}$ & $\begin{array}{l}\rightarrow \text { MPS } \\
\rightarrow \text { Chromosomal } \\
\text { abnormalities or } \\
\text { genetic mosaicism } \\
\rightarrow \text { Lowe syndrome } \\
\rightarrow \text { PTEN- } \\
\text { associated } \\
\text { disorders } \\
\rightarrow \text { Histiocytosis } \\
\rightarrow \text { Branched-chain } \\
\text { amino acid } \\
\text { disorders }\end{array}$ \\
\hline
\end{tabular}

Figure 4. Continued.

homogenize across different diseases. WMSAs that manifest in different locations can eventually progress to confluence and invade most of the brain tissue. In these scenarios, properties that the algorithm requires (e.g. primary WMSA location, isolation and signal intensity) are lost, and the algorithm is not diagnostically valuable. These limitations stress the need for early MRI scans in the clinical implications of the algorithm, which is supported by these data.

Since the day-to-day professional responsibilities of radiologists do not include determining differential diagnoses but rather solely identifying WMSA patterns on MRI scans, relying on radiologists alone may be insufficient for clinical applications. The algorithm would likely display a higher utility if other clinicians such as genetic/metabolic/neurological specialists also provided ratings. Realistic usage of this algorithm would involve radiologists concluding a category of WMAs with commonly manifesting WMSAs on cranial MRI scans and other clinicians using clinical abstractions to narrow this category into differential diagnoses, thus close collaboration between neuroradiologists and genetic/metabolic/neurologist physicians is helpful.

Without MRI emphasis, some WMAs may require sequential molecular testing using targeted $\mathrm{LD} / \mathrm{LE}$ gene panels that are commercially available. If these panels 
are negative or inconclusive, whole exome or genomic sequencing may be used and these tests can be a significant expense to the patient and health care systems. In the current fiscal climate, a thorough study of cranial MRI scans by a team of radiologists and clinicians (metabolic geneticists, neurologists, etc.) may help in streamlining the requirement of molecular/biochemical tests. ${ }^{18}$ Average pricing estimates at our hospital suggest that cranial MRI per patient costs $<$ CAD\$1000 compared to the comprehensive LD panels and exome sequencing, which cost approximately CAD $\$ 3000-5000$ and CAD\$5000, respectively.

The algorithm in this study promotes awareness among radiologists and other specialists of the different aspects of WMAs via MRI. Despite recording low validity statistics, the algorithm should be viewed in the context of the patient sample, the inclusion criteria, and its limitations. Had the inclusion criteria demanded for patients with multiple sequential MRI scans that were a minimum of six months apart, the algorithm would have been more applicable. Similarly, the reduced utility of the algorithm in wellmanaged and end-stage WMAs should be considered as a limitation. Improvements to this study methodology would include a larger patient sample and the inclusion of more radiologists as well as other clinical specialists. The addition of clinicians and $>2$ radiologists would allow for better quantification of inter-rater reliability and reality simulation. The centre where this study was conducted was a tertiary care centre, and it would be of interest to see how future studies can be performed at other health care levels with varying resources. As a pilot study, the patient sample was significantly heterogeneous in its different patient diagnostic time points and range of WMAs. Future diagnostic test values will be refined if narrowed down to a more specific sample type, and having more non-specific cases will produce more precise specificity and NPV values.

\section{Conclusion}

It should be noted that this MRI algorithm is currently the most systematic way to analyze MRI scans in the context of WMAs. In some scenarios, MRI analysis may result in a non-specific diagnosis for which differential diagnoses are difficult to pinpoint without full molecular testing. However, even with non-specific conclusions, MRI pattern analyses are useful in excluding many disorders that may not need to be tested for. ${ }^{10}$ Thorough MRI review can assist in deciding which specific molecular tests to follow-up with, which can have decreased financial burden to the health care model and benefits to patient comfort. This pilot study suggests that this algorithm has significant clinical implications, but much remains to be seen in terms of its shortcomings, applications in clinical situations, potential improvements and eventual introduction into clinical settings.

\section{Acknowledgements}

We would like to extend our thanks to the patients and their families for their part in this study. We also thank Mr W Johnson in Medical Imaging for processing and anonymizing patient MRI scans.

Electronic database information: URLs used in preparation of this article are Locus Link, National Center for Biotechnology Information, National Library of Medicine (Bethesda, MD), 1999, https://www.ncbi.nlm.nih.gov/ pubmed; Online Mendelian Inheritance in Man, OMIM ${ }^{\mathrm{TM}}$, Center for Medical Genetics, Johns Hopkins University (Baltimore, MD); and National Center for Biotechnology Information, National Library of Medicine (Bethesda, MD), 1999, http://www.ncbi.nlm.nih.gov/omim/.

\section{Funding}

This work was supported by the Children's Hospital Foundation, London, Ontario, and Western University, London, Ontario. The Children's Hospital Foundation, London, Ontario, has funded the student stipend for student researcher RD.

\section{Conflict of interest}

The authors declared no potential conflicts of interest with respect to the research, authorship, and/or publication of this article.

\section{References}

1. Hasegawa M, Houdou S, Mito T, et al. Development of myelination in the human fetal and infant cerebrum: A myelin basic protein immunohistochemical study. Brain Dev 1992; 14: 1-6.

2. Kumar S, Mattan NS and de Vellis J. Canavan disease: A white matter disorder. Ment Retard Dev Disabil Res Rev 2006; 12: 157-165.

3. Janardhan V and Bakshi R. Quality of life in patients with multiple sclerosis: The impact of fatigue and depression. J Neurol Sci 2002; 205: 51-58.

4. de Baulny HO, Benoist JF, Rigal O, et al. Methylmalonic and propionic acidaemias: Management and outcome. $J$ Inherit Metab Dis 2005; 28: 415-423.

5. Muñoz A, Mateos F, Simón R, et al. Mitochondrial encephalomyopathies in children. Part I: Conventional MR imaging findings. Curr Med Imaging Rev 2009; 5: 85-99.

6. Heim P, Claussen M, Hoffmann B, et al. Leukodystrophy incidence in Germany. Am J Med Genet 1997; 71: 475-478.

7. Bonkowsky JL, Nelson C, Kingston JL, et al. The burden of inherited leukodystrophies in children. Neurology 2010; 75: 718-725.

8. Wenger DA. Krabbe disease. In: Adam MP, Ardinger HH, Pagon RA, et al. (eds) GeneReviews ${ }^{\circledR}$. Seattle, WA: University of Washington, Seattle, 2000. https://www. ncbi.nlm.nih.gov/books/NBK1238 (accessed 2 March 2018).

9. van der Knaap MS and Valk J. Magnetic resonance of myelination and myelin disorders. 3rd ed. Berlin, Heidelberg, New York: Springer, 2005, pp.37-65.

10. Parikh S, Bernard G, Leventer RJ, et al. A clinical approach to the diagnosis of patients with leukodystrophies and genetic leukoencephelopathies. Mol Genet Metab 2015; 114: 501-515. 
11. Van Haren K, Bonkowsky JL, Bernard G, et al. Consensus statement on preventive and symptomatic care of leukodystrophy patients. Mol Genet Metab 2015; 114: 516-526.

12. Vanderver A, Hussey H, Schmidt JL, et al. Relative incidence of inherited white matter disorders in childhood to acquired pediatric demyelinating disorders. Semin Pediatr Neurol 2012; 19: 219-223.

13. van der Knaap MS, Breiter SN, Naidu S, et al. Defining and categorizing leukoencephalopathies of unknown origin: MR imaging approach. Radiology 1999; 213: 121-133.

14. Schiffmann R and van der Knaap MS. Invited article: An MRI-based approach to the diagnosis of white matter disorders. Neurology 2009; 72: 750-759.
15. Vanderver A, Prust M, Tonduti D, et al. Case definition and classification of leukodystrophies and leukoencephalopathies. Mol Genet Metab 2015; 114: 494-500.

16. Lalkhen AG and McCluskey A. Clinical tests: Sensitivity and specificity. Contin Educ Anesth Crit Care Pain 2008; 8: 221-223.

17. McHugh ML. Interrater reliability: The kappa statistic. Biochem Med 2012; 22: 276-282.

18. Richards J, Korgenski EK, Srivastava R, et al. Costs of the diagnostic odyssey in children with inherited leukodystrophies. Neurology 2015; 85: 1167-1170. 